\title{
Photometry of R Coronae Borealis
}

\author{
J. D. FERNIE \\ (with Vicki Sherwood and David L. DuPuy)
}

(Toronto)

Photoelectric photometry of $\mathrm{R} \mathrm{CrB}$ was obtained during the years 1966-71. During this time the star emerged from a prolonged minimum, showing a change in colour indices which was very like those of the supergiant sequence between $G 8$ and $F 8$ if allowance is made for an ultraviolet excess and some interstellar reddening. Superimposed on this rise towards maximum was a sharp minimum, during which the $\mathrm{V}$ magnitude was observed to decline through more than 3 magnitudes without any corresponding reddening in the colour indices. Throughout the emergence from the extended minimum the star showed irregular fluctuations of up to 0.2 mag. on a timescale of weeks.

Detailed observations near maximum light showed that $\mathrm{R} C r B$ exhibits a sinusoidal variability of period about $44^{\mathrm{d}}$ and amplitude about $0.15 \mathrm{mag}$. The light-curve is somewhat irregular and suggests RV Tauri-like behaviour. The B-V colour-curve seems irregular with an amplitude of about 0.04 mag., while the radial velocity at maximum is also slightly variable by 4 or $5 \mathrm{~km} / \mathrm{sec}$.

Assuming the star to be pulsating at maximum, the $44^{\mathrm{d}}$ period may be combined with SEARLE's spectroscopic determination of its surface gravity to indicate a mass of about $2 \mathrm{M}_{\odot}$ and a radius of about $100 \mathrm{R}_{\odot}$.

[The complete details of this work will appear shortly in the Astrophysical Journal.]

\section{Discussion to the paper of FERNIE}

SCHUMANN: What is the precision of your estimates of the U-B colour indices in your first slide? There are 2 points om 1 higher than the constant level during the light minimum.

FERNIE: In those earlier observations taken without use of a comparison star, the general precision of U-B is about \pm omo3.

LLOYD EVANS: RY Sgr shows complicated colour and spectral changes during a deep minimum; emission lines and reddening by the ejected cloud effect the colours. Observations near maximum show a 39 day pulsation (semi-regular), visual amplitude $=0$ m, 4 , with colour and velocity changes similar to those in a W Vir star.

\section{Models for R Coronae Borealis Stars}

\section{P. BIERMANN and R. KIPPENHAHN (Göttingen)}

If we believe in the spectral analysis of the atmospheres of $\mathrm{R} \mathrm{CrB}$ stars these objects should have a pure helium envelope slightly enriched in carbon (SEARLE 1961, DANZIGER 1965). However, they cannot be homogeneous helium stars since in that case they should be on the helium main sequence in the Hertzsprung-Russell-diagram, whereas they are found in the region of late supergiants. Their internal chemical structure must therefore be more complicated than that of a homogeneous helium star. From the viewpoint of stellar evolution the next degree of complexity would be to have a carbon-oxygen core. 